\title{
Planning for small cells in a cellular network: Why it is worth it
}

\author{
Rajasekhar Sappidi, Sajjad Mosharrafdehkordi, Catherine Rosenberg, and Patrick Mitran \\ Dept. of Electrical and Computer Engineering, University of Waterloo \\ Waterloo, Ontario, Canada \\ Email: \{rrsappid, smosharr, cath, pmitran\}@uwaterloo.ca
}

\begin{abstract}
Most of the literature on heterogeneous cellular networks is focused on analyzing them as a single macro cell embedded with small cells. In this paper, we take a global perspective and analyze the effect of deploying small cells on the performance of a network comprising several macro cells. We identify potential locations for low-power base-stations based on the coverage patterns of the macro cells and propose three schemes for placing the small cells. Using the model recommended by 3GPP, we show that by judiciously installing just two small cells for every macro base-station at these locations and allocating separate resources to all the small cells on a global level, we can increase the performance of the network significantly $(\sim 45 \%)$. An added benefit of our schemes is that we can switch off the macro base-stations at night (when the number of active users is low) and significantly reduce their operation cost.
\end{abstract}

\section{INTRODUCTION}

Cellular networks have witnessed an overwhelming growth in data traffic in the last decade and this trend is expected to continue in the future [1]. Thus, there is a need to increase the data capacity of these networks. For a long time, the state-ofthe-art architecture for a cellular network has been to deploy only one type of large base-stations, known as the macro base-stations (MBS), regularly over a large area, resulting in a homogenous cellular network. However, this approach is no longer sufficient to meet the exponentially growing demand for network throughput. Deploying additional lowpower base-stations (e.g., pico base-stations (PBS), femto basestations (FBS), etc.) is one of the most promising approaches to increase the throughput of the network in a cost (and energy) effective manner [2]. However, this now results in a heterogeneous cellular network $(\mathrm{HCN})$ structure. Small cell is the generic term used for base stations that are deployed by the operator and have either a wired (e.g., pico) or a wireless (e.g., relay) link to the backhaul network. There has been a large research thrust on $\mathrm{HCNs}$ which has established that the deployment of small cells indeed improves the throughput of cellular networks. However, most of this research is focused on the performance evaluation of a single macro cell embedded with small cells. Unlike this, in this paper, we consider an entire network consisting of several macro cells and propose three different schemes for the small cell deployment. We then evaluate the impact of these small cells on the overall throughput capacity of the network. For this evaluation, we need to find the joint optimal resource allocation (division of resources between the macro cells and the small cells, RA for short), user association (determines which users are served by which BSs, UA for short) and scheduling (the fraction of time a BS serves a user). Computation of these aspects of a $\mathrm{HCN}$ jointly and optimally requires solving a very large nonlinear integer program to optimality. We handle this problem by first obtaining an upper bound on its objective by relaxing the integer constraints. We then use simple UA rules (see Section III for more details) to obtain a feasible solution whose objective is within $10 \%$ of the upper bound.

Location planning and selection for small cells is one of the least investigated aspects of HCNs. It is often assumed that they are either symmetrically placed in the macro cell [3], [4] or that they are uniformly distributed in the macro cell [5] or that they are placed close to the hot spots [6], [3]. Although, placing the small cells within the interior of the macro cell seems natural, it might be more beneficial to place them at the intersections of the macro cells where the coverage is weak and the interference is strong. To the best of our knowledge, these type of location placements for small cells where they overlap with more than one macro cell have never been considered in the literature. In this paper, we identify two sets of such potential locations for small cells within a homogenous cellular network and propose three different schemes of installing small cells based on these locations. Also, unlike most of the literature, we assume that the resource allocation to the small cells is done at the network level globally. We also do not consider the scenario with hot spots and assume that the users are uniformly distributed in the network.

In this paper, we consider proportional fairness as the fairness criteria [7], [3]. Thus, the geometric mean (GM) throughput of all the users is the performance metric of a given network configuration (i.e., resource allocation, user association and scheduling) and we are interested in maximizing it. Using this metric, we show (via numerical results on a large network) that surprisingly significant performance gains ( $\sim 45 \%$ ) can be achieved by judiciously deploying just two small cells for every macro cell. For this numerical evaluation, we have considered the model recommended by 3GPP [8].

Our main contributions are as follows.

1) We propose three placement schemes for small cells and show that judiciously installing just two small cells for each macro cell (and allocating separate resources to the small cells on the network level) is enough to achieve a large gain $(\sim 45 \%)$ in the performance of the network. Unlike much of the literature, we evaluate the performance of the network from a global perspective and not just for a single macro cell.

2) We show that the performance of simple user association rules like best SINR and small cell first (SCF) is within $10 \%$ of the upper bound on the performance.

3) An additional benefit of our small cell placement and resource allocation schemes is that it is possible 
to switch off the MBSs at nights. In other words, the small cell planning and placement proposed here is enough to maintain connectivity under low traffic conditions. This is important as MBSs consume a significant amount of power for their operation.

The rest of this paper is organized as follows. In Section II, related literature is presented. In Section III, the system Model and the three proposed schemes are described. In Section IV, we mathematically formulate the problem. In Section V, numerical results are provided. Section VI concludes the paper.

\section{RELATED WORK}

There is an extensive literature on various aspects of heterogeneous cellular networks. In particular, in [9], [3], [4], it has been shown that installing low-power base stations such as pico base-stations, increases the throughput of the cellular network. However, these types of performance evaluation are for a single macro cell with small cells placed either symmetrically around the MBS or close to the hotspots. In [5], the small cells are assumed to be uniformly distributed within the macro cell while in [6], the location of the small cells are optimized within a macro cell. In these works, it is also assumed that the resources allocated to the small cells belongs to the macro cell they are installed in. Unlike these works, in this paper, we consider a HCN comprised of several macro cells and assume that the resource allocation to the small cells is done at the network level globally.

HCNs have also been analyzed using stochastic geometry techniques [10], [11]. There, the BSs are randomly distributed in the network area. However, these techniques do not shed any light on the best locations for the small cells. In [12], an optimization problem is proposed that can choose the best locations for MBSs and small cells from among a given set of potential locations while in [13], a Gibbs sampling based optimization is used to find the best locations for small cells. Without using any type of optimization, in this paper, based on the interference and coverage patterns, we identify potential locations for small cells in a homogenous cellular network. We show that installing small cells at these locations and allocating exclusive resources globally to the small cells significantly improves the performance of the network.

\section{SySTEM MODEL}

First, we introduce the baseline system which we use as the benchmark for our schemes.

\section{A. Baseline System}

We consider an OFDM-based homogeneous cellular network as our baseline system. In this network, we assume that there are $M$ macro base-stations (MBS) each equipped with three directional antennas, a user density of $\rho_{u}$ users per cell and $T$ sub-channels, each of bandwidth $b$. We consider only the downlink traffic in this system and assume that the BSs transmit all the time in all the channels assigned to them. In Fig. 1, we provide a pictorial depiction of this system.

We assume that a frequency reuse factor of 3 is used and $T / 3$ sub-channels are exclusively assigned to each of the directional antennas. We quantify the performance of this

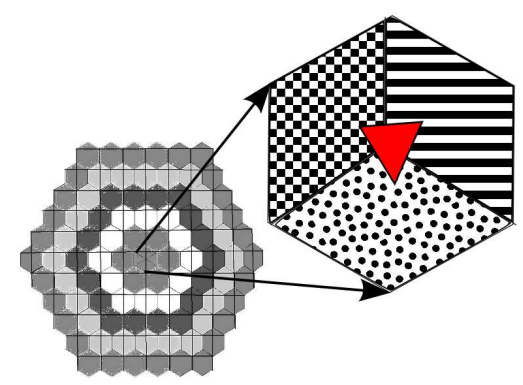

Fig. 1: A macro cell of the baseline system. Each sector is served by one of the directional antenna of the MBSs (represented by the large triangle).

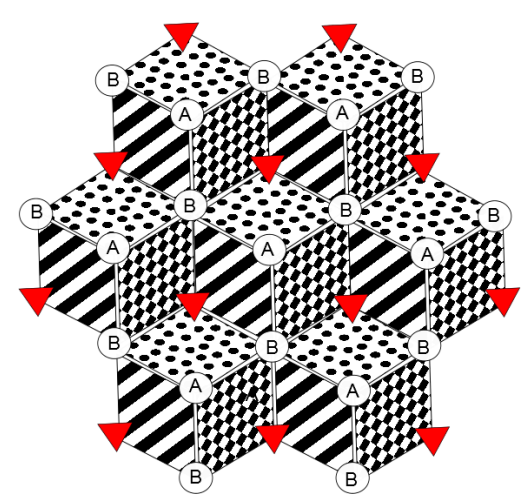

Fig. 2: A part of the cellular network. Here locations A and $\mathrm{B}$ are conducive for installing small cells.

network in terms of the geometric mean (GM) throughput of all the users. We are interested in measuring the impact of deploying low-power BSs with omnidirectional antennas on the performance of this system.

\section{B. Placement of small cells}

As shown in Figure 2, we have identified two types of potential locations for the deployment of the small cells. At these locations, the coverage is weak and/or the interference can be strong. At locations of type A, three sectors belonging to three different bands meet while at locations of type B, six sectors meet such that there are two sectors from each band. Since, every point of intersection is shared by three hexagonal cells, it is easy to see that, for a large network and neglecting edge effects, there is one point of type A and one point of type $\mathrm{B}$ for each MBS.

Depending on the type of locations chosen for the installation of the small cells, we define three different schemes.

- Scheme A: Install the small cells only at locations of type A.

- Scheme B: Install the small cells only at locations of type B.

- Scheme C: Install them at both locations of type A as well as type B. 
Note that in Scheme A and Scheme B, we install one small cell per MBS and in Scheme C, we install two small cells per MBS. We next discuss the resource allocation used for each of these 3 schemes.

\section{Global Resource Allocation}

We assume a simple global resource allocation scheme in which $K$ channels are assigned to the small cells and $T-K$ are assigned to the macro-base stations. Note that the small cells use omni-directional antennas and utilize the $K$ channels with a reuse factor of 1 globally. We assume that the $T-K$ sub-channels assigned to the MBS are equally divided among its three directional antennas. So, every directional antenna of the MBS is assigned $(T-K) / 3$ sub-channels.

\section{Power Allocation}

Let the transmit power of the MBS be $P_{\text {MBS }}$ and that of a small cell BS be $P_{\mathrm{SC}}$. We assume that $P_{\mathrm{MBS}}$ is equally divided among the three directional antennas of the MBS. We also assume that the power is equally divided among all the subchannels assigned to a base-station. Thus, the transmit power per subchannel is given as follows,

$$
P_{i}= \begin{cases}\frac{P_{\mathrm{MBS}}}{T-K} & \text { if } i \text { is a MBS } \\ \frac{P_{\mathrm{SC}}}{K} & \text { if } i \text { is a small cell }\end{cases}
$$

Note that this means that the interference is a function of $K$ as will be seen in the next subsection.

\section{E. The Channel Model}

We model the channel gain, $g_{j u}$, between BS $j$ and user $u$ as, $g_{j u}=D_{j u} \times G_{j u} \times P L_{j u}$, where $P L_{j u}$ is the path-loss, $G_{j u}$ is the log normal shadowing, and $D_{j u}$ is the directive gain pattern of the antenna. For the path-loss and the directive gain pattern of the antenna, we consider the model recommended by 3GPP [8]. According to this recommendation, the path-loss, $P L_{j u}$, follows the model given in Table I. For the directive gain pattern of a directional antenna, they recommend $D_{j}(\theta)=$ $-\min \left\{12\left(\frac{\theta}{\theta_{3 \mathrm{~dB}}}\right)^{2}, A_{m}\right\} \mathrm{dB}$, where $\theta$ is the angle made by the user position with the broadside direction of the antenna, $\theta_{3 \mathrm{~dB}}$ is the $3 \mathrm{~dB}$ beamwidth $\left(\theta_{3 \mathrm{~dB}}=70\right.$ degrees $)$ and $A_{m}=20$ is the maximum attenuation in $\mathrm{dB}$ which the signal experiences in the sidelobes of the antenna. The directive gain pattern of an omnidirectional antenna is assumed to be $0 \mathrm{dBi}$ for every angle. We assume that the shadowing $G_{j u}$ follows a log normal distribution with mean 0 and standard deviation of $8 \mathrm{~dB}$.

The SINR between BS $j$ and user $u$ is given as follows,

$$
\gamma_{j u}=\frac{g_{j u} P_{j}}{N_{0}+\sum_{i \in \mathcal{B}_{j}} g_{i u} P_{i}},
$$

where $P_{i}$ is the transmit power per subchannel of BS $i, N_{0}$ is the additive white Gaussian noise power and $\mathcal{B}_{j}$ is the set of all BSs that are transmitting on the same channels as BS $j$. Note that, since the transmit power $P_{i}$ is a function of $K$ (the number of subchannels assigned to the small cells), SINR is also a function of $K$. Let $\tilde{r}_{j u}$ be the data rate per subchannel received by user $u$ from $\mathrm{BS} j$. This is a function of the SINR received by user $u$ from $\mathrm{BS} j, \gamma_{j u}$, i.e., $\tilde{r}_{j u}=f\left(\gamma_{j u}\right)$. We assume that the function $f($.$) is the same for MBS and small$ cells. We consider the piecewise linear function recommended by 3 GPP for this (per subchannel) rate function (see Section $\mathrm{V}$ for more details).

\section{F. User Association}

A user association (UA) policy determines the BS to which a given user connects with. We consider the following two simple user association (UA) rules. They are

- Best SINR: Users associates with the BS that provides the maximum SINR.

- Small-cell First (SCF): Under this rule, a user associates with the small cell that gives the maximum SINR provided that this is greater than a given threshold $\beta$. If no small cell provides SINR greater than $\beta$, it associates with the BS that provides the maximum SINR.

Note that as $\beta \rightarrow \infty$, SCF rule converges to best SINR rule. Note also that our SCF rule is slightly different from the one considered in the literature [4], [3], where the user connects only to one of the macro BSs if there is no small cell that can provide a SINR greater than the given threshold. In the next section, we give a mathematical formulation of our problem.

\section{PRoblem Formulation}

In this section, we first mathematically formulate the problem for a general heterogeneous cellular network as a joint optimal scheduling, resource allocation and user association problem.

Let $\mathcal{B}$ be the set of all the BSs (MBSs + small cells) in the network and $\mathcal{B}_{i}$ be the set of all BSs that are assigned the same set of subchannels as BS $i$. Let $\mathcal{N}$ be the set of all users in the network and let $\lambda_{u}$ be the data rate received by user $u$. We assume that the users are greedy and are interested in maximizing their throughput. Thus, there is a need to ensure fairness in the network and we consider the proportional fair criteria [7], [3], which requires maximizing the sum of the logarithms of the throughputs.

Let $x_{i u}$ be the user association variable which is a binary variable that is 1 if user $u$ is associated with $\mathrm{BS} i$ and otherwise 0 . Let $\alpha_{i u}$ be the fraction of time BS $i$ transmits to user $u$. Clearly, $\alpha_{i u}=0$ whenever $x_{i u}=0$. We can compute the geometric mean throughput of a given system by solving the following optimization problem $\mathcal{P}$ and also find the corresponding user association $\left(x_{i u}\right.$ 's), the schedule $\left(\alpha_{i u}\right.$ 's) and the number of sub-channels to be assigned to the small cells $(K)$. Note that when the given system is our baseline system, $\mathcal{B}$ consists of only the macro BSs and $K=0$.

$$
\begin{array}{r}
\mathcal{P}: \quad \operatorname{Max}_{\alpha, \mathbf{x}, K} \sum_{u} \log \left(\lambda_{u}\right) \\
\text { subject to } \\
\lambda_{u}=\sum_{i \in \mathcal{B}} \alpha_{i u} r_{i u}
\end{array}
$$


TABLE I: Path-loss model

\begin{tabular}{|c|c|c|c|c|}
\hline Transmitter & Link $(j, i)$ & Path-loss of the medium $\left(\phi_{j u}\right)(\mathrm{dB})$ & Antenna gain $\left(A G_{j}\right)(\mathrm{dB})$ & Losses $\left(\zeta_{j}\right)(\mathrm{dB})$ \\
\hline MBS & $(j, u)$ & $128.1+37.6 \log _{10}\left(\frac{d_{j u}}{1000}\right), d_{j u} \geq 35 m$ & 15 & 20 \\
Small cell & $(j, u)$ & $140.7+36.7 \log _{10}\left(\frac{d_{j u}}{1000}\right), d_{j u} \geq 10 m$ & 5 & 20 \\
\hline \hline
\end{tabular}

$$
\begin{aligned}
r_{i u} & =N_{i} \times f\left(\gamma_{i u}\right) \\
\gamma_{i u} & =\frac{g_{i u} P_{i}}{N_{0}+\sum_{j: j \neq i, j \in \mathcal{B}_{i}} g_{j u} P_{j}} \\
P_{i} & = \begin{cases}\frac{P_{\mathrm{MBS}}}{T_{\mathrm{SC}}-K} & \text { if } i \text { is a MBS } \\
K & \text { if } i \text { is a small cell }\end{cases} \\
N_{i} & = \begin{cases}(T-K) / 3 & \text { if } i \text { is a MBS } \\
K & \text { if } i \text { is a small cell }\end{cases} \\
\sum_{u \in \mathcal{N}} \alpha_{i u} & \leq 1 \\
0 \leq \alpha_{i u} & \leq x_{i u} \\
\sum_{i \in \mathcal{B}} x_{i u} & =1 \text { and } \quad x_{i u} \in\{0,1\}
\end{aligned}
$$

Constraint (4) represents the total data rate received by user $u$. Eq. (5) computes the data rate between a user and a BS using the given (per subchannel) rate function, $f(\cdot)$. Equation (6) computes the SINR between the BSs and the users. Eqs. (7) and (8) compute the power per sub-channel and number of sub-channels at each BS, which depends on whether the BS is a macro BS or a small cell BS. Constraint (9) states that the sum of the fractions of scheduling times from a given BS cannot exceed 1. Constraint (10) ensures that the BS allocates scheduling time to a user only if it is associated with it and Constraint (11) ensures that a user associates with only one BS.

This optimization problem, $\mathcal{P}$, is a non-linear integer program and hence is very difficult to solve. There are three reasons for this.

1) The SINR constraint in Eq. (6) is non-convex (because of its dependence on $K$ ).

2) The constraint with rate function, i.e., Eq. (5), is also difficult to handle. We require either a closed form expression for $f(\cdot)$ that is convex or a method to enumerate all the rates and eliminate this constraint.

3) It is an integer program and is not tractable for large problem sizes.

A usual technique used to address the issue with the SINR constraint (6), is to fix $K$, the number of channels assigned to the small cells and compute the SINRs, $\gamma_{i u}$ 's, a priori. Given the SINRs and the rate function, $f(\cdot)$, we can compute the data rates available from every BS to every user and thus address the issue with the constraint in Eq. (5). With these two modifications, the problem transforms into a simpler non-linear integer program. However, it is still intractable in its current form. So, we relax the integer constraint on $x_{i u}$ 's and find an upper bound on the GM throughput. This relaxation allows the users to be associated with multiple BSs and requires that the traffic destined to a user be split and delivered via different BSs.

We also find feasible solutions by assuming that the user association (UA) variables, $x_{i u}$ 's, are determined by the simple UA rules given in Section III-F viz., best SINR and small-cell first (SCF). We define the envelope of these feasible solutions as the best feasible solution over all the considered UA rules at every $K$. In Section V, we present a comparison of the upper bound with this envelope of the GM throughput of the feasible solutions versus $K$, which shows that there are near-optimal and within $10 \%$ for all three schemes.

For the feasible solution case, since the data rates, $r$, i.e., $r_{i u}$ 's, are computed a priori by fixing $K$ and that the user association, $\mathbf{x}$, i.e., $x_{i u}$ 's is given, we can compute the geometric mean throughput of the system by solving the following optimization problem $\mathcal{P}(\mathbf{r}, \mathbf{x})$.

$$
\begin{aligned}
\mathcal{P}(\mathbf{r}, \mathbf{x}): \quad \operatorname{Max}_{\alpha} & \sum_{u} \log \left(\lambda_{u}\right) \\
\text { subject to } & \\
\lambda_{u} & =\sum_{i \in \mathcal{B}} \alpha_{i u} r_{i u} \\
0 \leq \alpha_{i u} & \leq x_{i u} \quad \forall i \in \mathcal{B} \quad \forall u \in \mathcal{N} \\
\sum_{u \in \mathcal{N}} \alpha_{i u} & =1
\end{aligned}
$$

This is a non-linear program. However, the nature of its solution is known and it can be easily computed using the following lemma, which is a specialized version of Lemma 1 in [7].

Lemma 1: Given the resource allocation parameters (the number of sub-channels allocated, the transmit power on each sub-channel and the user association) and infinite backhaul capacity, under proportional fair scheduling a BS assigns equal proportion of time to all the users associated with it.

This lemma implies that local equal time scheduling (at every BS) solves our optimization problem, $\mathcal{P}(\mathbf{r}, \mathbf{x})$ and thus we have an efficient technique to compute its optimal solution. In the next section, we present numerical results obtained by solving this problem (using the above Lemma) for a large number of random network realizations.

\section{Numerical Results}

We consider a large cellular network with 91 macro cells (or 5 tiers of hexagonal cells around the central cell). We consider two different scenarios, viz., urban and rural. In the urban scenario, the inter-site distance (ISD) between the macros is $500 \mathrm{~m}$ while in the rural case, it is $1732 \mathrm{~m}$ [8]. For both these scenarios, we assume that the users are uniformly 
TABLE III: Modulation and coding schemes - LTE

\begin{tabular}{|c|c|c|c|c|c|c|c|c|c|c|c|c|c|c|c|}
\hline $\mathrm{R}(\mathrm{dB})$ & -6.5 & -4 & -2.6 & -1 & 1 & 3 & 6.6 & 10 & 11.4 & 11.8 & 13 & 13.8 & 15.6 & 16.8 & 17.6 \\
\hline
\end{tabular}

TABLE II: Physical Layer Parameters

\begin{tabular}{|c|c|c|c|}
\hline Noise Power & $-174 \frac{\mathrm{dBm}}{\mathrm{Hz}}$ & $T_{\text {subframe }}$ & $1 \mathrm{~ms}$ \\
\hline $\mathrm{P}_{\mathrm{Sc}}$ & $30 \mathrm{dBm}$ & $\mathrm{P}_{\text {macro }}$ & $46 \mathrm{dBm}$ \\
\hline UE Ant. Gain & $0 \mathrm{~dB}$ & Sub-channel Bandwidth & $180 \mathrm{KHz}$ \\
\hline Shadowing s.d. & $8 \mathrm{~dB}$ & User Noise Figure & $9 \mathrm{~dB}$ \\
\hline Penetration Loss & $20 \mathrm{~dB}$ & M (Number of sub-channels) & 100 \\
\hline Macro Ant. Gain & $15 \mathrm{dBi}$ & Pico Ant. Gain & $5 \mathrm{dBi}$ \\
\hline SC $_{\text {ofdm }}$ & 12 & SY $_{\text {ofdm }}$ & 14 \\
\hline
\end{tabular}

distributed with a density of $\rho_{u}=25$ users per cell. Note that since the rural cell has 10 times larger area, its density of users per unit area is 10 times smaller than that of a urban cell. We have generated 100 random realizations of the user locations for each scenario.

The physical layer parameters used in our computations are given in Table II [8]. The typical transmit power of the MBS is $46 \mathrm{dBm}$ and that of the small cell is $30 \mathrm{dBm}$. We assume that there are a total of $T=99$ subchannels which can be divided among the macro and the small cells. For the (per subchannel) rate function, we use the piecewise linear mapping (recommended by 3GPP), which is given by $\tilde{r}_{j u}=$ $\frac{\mathrm{SC}_{\text {offm }} \mathrm{SP}_{\text {offm }}}{T_{\text {sufframe }}} e_{l}$, where $e_{l}$ is the efficiency (bits/symbols) of the corresponding SINR threshold level $l, \mathrm{SC}_{\text {ofdm }}$ is the number of data subcarriers per sub-channel bandwidth, $\mathrm{SY}_{\text {ofdm }}$ is the number of OFDM symbols per subframe, and $T_{\text {subframe }}$ is the subframe duration in time units. The mapping of $e_{l}$ to SINR levels is given in Table III.

Recall that in problem $\mathcal{P}(\mathbf{r}, \mathbf{x})$, the input rates $\mathbf{r}$ are known by fixing $K$ and the user association, $\mathbf{x}$, is determined by either the best SINR rule or the SCF rule. For each of the 100 random realizations in both the scenarios, we solved the problem $\mathcal{P}(\mathbf{r}, \mathbf{x})$ using Lemma 1, for all three proposed schemes and the baseline system, for every $K$ from $K=3$ to $K=96$ and for both the user association rules, best SINR and SCF (for the baseline system, we have used the best SINR user association rule). For SCF, we solved for 15 different $\beta$ 's, taken from the thresholds row in Table III. Thus, we have 16 different user association rules.

In Fig. 3, for the urban scenario, we have plotted the upper bound on the GM throughput obtained by solving the relaxed problem versus the best GM throughput (or the envelope) due to the $16 \mathrm{UA}$ rules considered, for all three schemes. We note that the performance of the best GM throughput (for each $K$ ) of our simple UA rules is within $10 \%$ of the upper bound. We also note from Fig. 3 that Scheme C with two small cells per MBS performs the best. When we can install only one small call per MBS, our results show that Scheme B is preferable over Scheme A.

Next, we computed the percentage gain in the geometric mean $(\mathrm{GM})$ throughput of every scheme with respect to the baseline GM and averaged it over the 100 random realizations (for every $K$ and the 16 user association rules). We repeated this for 7 different transmit powers of the small cells BS.

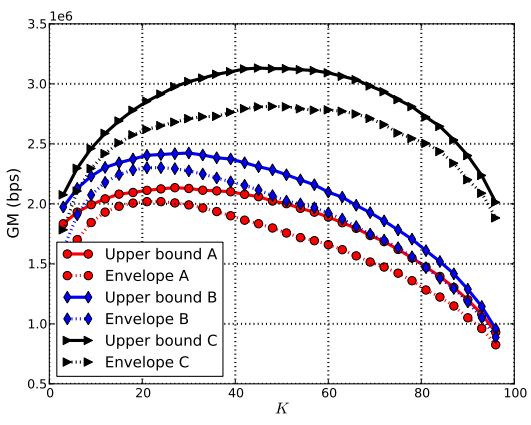

Fig. 3: Upper bound and the best GM throughput using our UA rules (labelled as the envelope) vs $\mathrm{K}$ for the urban scenario, $\rho_{u}=25$ and $P_{\mathrm{SC}}=30 \mathrm{dBm}$ for all three schemes

For every considered transmit power of the small cell, we have computed the best possible gain for any $K$ and any user association rule considered and plotted it versus $P_{\mathrm{SC}}$ in Fig. 4 for both the urban and rural scenarios. From these plots, we can infer that by judiciously installing small cells according to Scheme C, we can obtain close to $45 \%$ gain in the geometric mean throughput of the system. We have also computed these results with a higher density of users $\left(\rho_{u}=50\right.$ users per cell). We observed that these results are also similar to the ones presented with a slight increase in the gains.

Another important observation from these results is that in the rural scenario, the small cells have to transmit at a much higher power to obtain a significant gain in performance, while for the urban scenario, the typical small cell transmit power of $30 \mathrm{dBm}$ is sufficient.

In Fig. 5, we show a comparison of the gains of the best SINR UA and the envelope of the best (for each $K$ ) 15 SCF user associations for the urban scenario. From this plot, we conclude that the best performance of the SINR user association rule is not far from the best performance of the SCF user associations.

MBSs have a significantly high energy cost of operation. We could substantially reduce this cost if we could afford to switch them off for a few hours every day when the number of active users is low. The proposed Scheme $\mathrm{C}$ provides a method to do this. In Fig. 6, we plot the percentage of uncovered area (as a function of $K$, when the MBSs are switched off and the small cells are deployed according to Scheme C. It is clear from this plot that the percentage of uncovered area is less than $2 \%$ when $K=45$, which implies that we can afford to switch off the MBSs during night time. Thus, Scheme C provides an added benefit that results in substantial savings in 


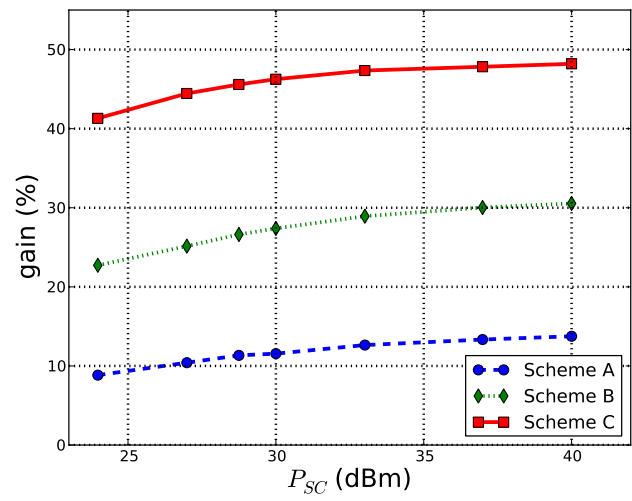

(a) Urban Scenario - ISD $=500 \mathrm{~m}$

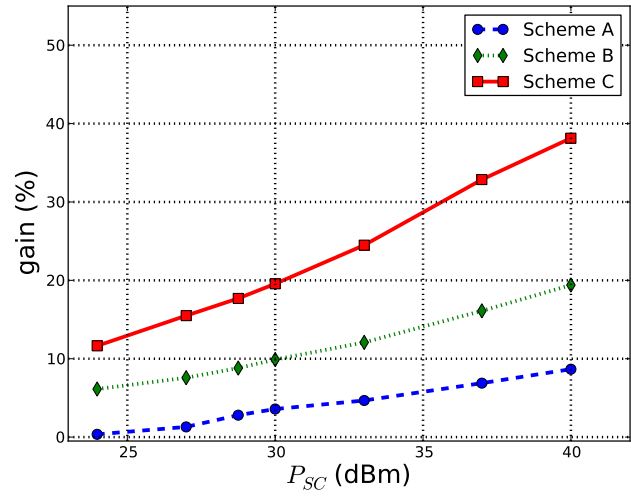

(b) Rural Scenario - ISD $=1732 \mathrm{~m}$

Fig. 4: Best gains (of best sinr and $15 \mathrm{SCF}$ UA rules) vs $P_{S C}$ for the three proposed schemes of small cell placement, $\rho_{u}=25$

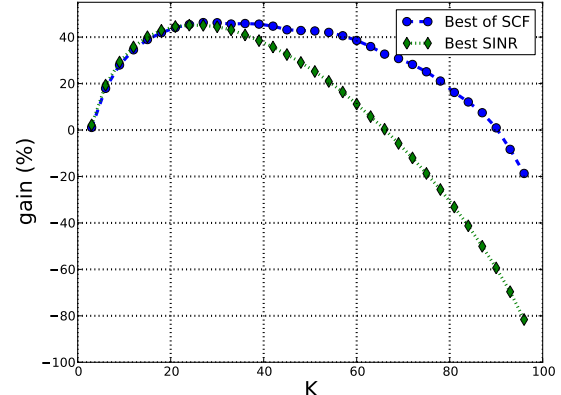

Fig. 5: Gains due to best SINR vs the best of SCF user associations for ISD $=500 \mathrm{~m}, \rho_{u}=25, P_{\mathrm{SC}}=30 \mathrm{dBm}$

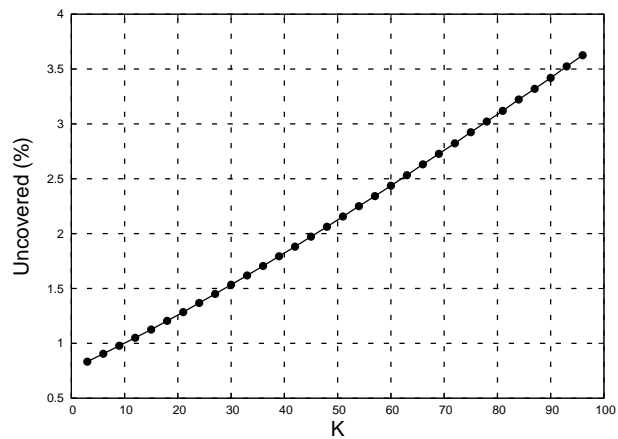

Fig. 6: \% of uncovered area with just small cells in Scheme $\mathrm{C}$ versus $K$ (urban scenario, $P_{\mathrm{SC}}=30 \mathrm{dBm}$ )

terms of the overall power consumption and cost of operation.

\section{CONCLUSION}

In this paper, we investigated the deployment of small cells in a cellular network from the overall network point of view. We have showed that judiciously installing two small cells per macro cell at the appropriate locations is enough to achieve a surprisingly significant gain of $\sim 45 \%$ in the performance of the network. We have used simple user association rules to achieve these gains and also showed that these user association rules give a GM throughput that is within $10 \%$ of the upper bound. We have also showed that an added benefit of our Scheme C is that we can afford to switch off the MBSs during night and reduce their operating cost.

\section{REFERENCES}

[1] UMTS Forum. Mobile traffic forecasts, 2010-2020 report, report number 44. January 2011.

[2] A. Damnjanovic, J. Montojo, Y. Wei, T. Ji, T. Luo, M. Vajapeyam, T. Yoo, O. Song, and D. Malladi. A survey on 3gpp heterogeneous networks. Wireless Communications, IEEE, 18(3):10-21, 2011.

[3] D. Fooladivanda and C. Rosenberg. Joint resource allocation and user association for heterogeneous wireless cellular networks. Wireless Communications, IEEE Transactions on, 12(1):248-257, 2013.

[4] J. Ghimire and C. Rosenberg. Resource allocation, transmission coordination and user association in heterogeneous networks: A flowbased unified approach. Wireless Communications, IEEE Transactions on, 12(3):1340-1351, 2013.

[5] Q. Li, R.Q. Hu, Y. Xu, and Y. Qian. Optimal fractional frequency reuse and power control in the heterogeneous wireless networks. Wireless Communications, IEEE Transactions on, 12(6):2658-2668, 2013.

[6] H. Shimodaira, G.K. Tran, S. Tajima, K. Sakaguchi, K. Araki, N. Miyazaki, S. Kaneko, S. Konishi, and Y. Kishi. Optimization of picocell locations and its parameters in heterogeneous networks with hotspots. In Personal Indoor and Mobile Radio Communications (PIMRC), 2012 IEEE 23rd International Symposium on, pages 124129, 2012.

[7] L. Li, M. Pal, and Y.R. Yang. Proportional fairness in multi-rate wireless lans. In INFOCOM 2008. The 27th Conference on Computer Communications. IEEE, pages -, 2008.

[8] 3GPP-TSG-RAN-WG1. Evolved universal terrestrial radio access (eutra). Tech. Rep. TR 36.814, 2010.

[9] D. Fooladivanda, A. Al Daoud, and C. Rosenberg. Joint channel allocation and user association for heterogeneous wireless cellular networks. In Personal Indoor and Mobile Radio Communications (PIMRC), 2011 IEEE 22nd International Symposium on, pages 384390, 2011.

[10] H. S. Dhillon, R. K. Ganti, F. Baccelli, and J. G. Andrews. Coverage and ergodic rate in k-tier downlink heterogeneous cellular networks. In Proc. Allerton Conference on Communication, Control, and Computing, September 2011. 
[11] H. S. Dhillon, R. K. Ganti, F. Baccelli, and J. G. Andrews. Modeling and analysis of k-tier downlink heterogeneous cellular networks. IEEE Journal on Sel. Areas in Comm., April 2012.

[12] W. Zhao and S. Wang. Cell planning for heterogeneous cellular networks. In Wireless Communications and Networking Conference (WCNC), 2013 IEEE, pages 1032-1037, 2013.

[13] X. Li, X. Tang, C. Wang, and X. Lin. Gibbs-sampling-based optimization for the deployment of small cells in $3 \mathrm{~g}$ heterogeneous networks. In Modeling Optimization in Mobile, Ad Hoc Wireless Networks (WiOpt), 2013 11th International Symposium on, pages 444-451, 2013. 